\title{
Pharmacologic therapy to induce weight loss in women who have obesity/overweight with polycystic ovary syndrome: a systematic review and network meta-analysis
}

\begin{abstract}
Background: Women with polycystic ovary syndrome (PCOS) are almost three times more likely to be obese than those without PCOS. However, we have no specific interventions to induce weight loss so far, and rely on drugs used to treat other symptoms of the syndrome or obesity in the general population.
\end{abstract}

Objective: To compare the effectiveness of metformin, inositol, liraglutide and orlistat to induce weight loss in women with PCOS and overweight/obesity.

Methods: A search was conducted using the MEDLINE, EMBASE, Pubmed and CENTRAL databases. Individually randomised, parallel group trials that evaluated the effects of these pharmacological treatments among adults or adolescents with PCOS and overweight/obesity, compared with a placebo or metformin group were considered eligible. Registration number: PROSPERO CRD 42017076625.

Results: Twenty-three trials reporting on 941 women were included in the network meta-analysis. The amount of weight lost differed significantly among the drugs (in descending order): liraglutide, orlistat and metformin. Liraglutide alone, liraglutide/ metformin, and metformin alone significantly reduced waist circumference but no 
change was found with orlistat. Data for WHR were only available for metformin, which had no significant effect.

Conclusion: Liraglutide appears superior to the other drugs in reducing weight and waist circumference.

Key words: overweight, obesity, polycystic ovary syndrome, pharmacologic therapy.

\section{Introduction}

The World Health Organization (WHO) has declared 'obesity is one of the greatest public health challenges of the 21 st century' ${ }^{\prime 1}$. Obesity is particularly a problem for women with PCOS as they have a 2.8 -fold increased prevalence (50\%-80\%) compared to women who do not have the syndrome ${ }^{2,3}$. In the original description of the syndrome by Stein and Leventhal in 1935, 60\% (three of the five women for whom habitus was reported) were obese ${ }^{4}$.

Unfortunately, this obesity, which is so common in women with PCOS, exacerbates symptoms of the syndrome ${ }^{5}$. Hyperinsulinaemia reduces circulating levels of sex hormone-binding globulin, increasing free testosterone, which inhibits follicular maturation with consequent menstrual irregularity and infertility. Insulin also augments the thecal androgen production responsible for acne and hirsutism ${ }^{6}$. In the longer term, 
hyperinsulinaemia increases the risks of diabetes (type II and gestational), coronary heart disease and endometrial cancer ${ }^{7-11}$.

Weight loss is therefore fundamental to the management of women with $\mathrm{PCOS}^{2,12}$. Aside from increasing the severity of the symptoms of PCOS, obesity reduces the effectiveness of fertility treatment (ovulation induction and in vitro fertilization) and increases the risk of pregnancy complications ${ }^{10,}{ }^{13}$. For these reasons, fertility treatment is withheld by the National Health Service (NHS) for those with a body mass index $(\mathrm{BMI})>30 \mathrm{~kg} / \mathrm{m}^{2}$. Although lifestyle management is the first line therapy in PCOS for prevention of weight gain and for weight loss ${ }^{14}$, the efficacy of lifestyle management for established obesity has been limited in PCOS in the general population ${ }^{15}$, and new approaches are needed ${ }^{16}$.Moreover, for some women with PCOS and obesity, lifestyle modifications are not effective, or for the women who cannot or are not willing to modify their lifestyle, the pharmacologic therapy to induce weight loss is also very important. Bariatric surgery can also effectively attenuates PCOS and its clinical symptomatology including hirsutism and menstrual irregularity in severely women with obesity ${ }^{17}$.The goal for weight reduction in women with PCOS is to improve insulin resistance, reduce hyperandrogenism and alleviate PCOS clinical severity ${ }^{15,18,19}$. However, because we do not understand the mechanisms responsible for obesity in women with this condition, we are forced to rely on drugs used to treat other symptoms of the syndrome or those licensed to treat obesity in the general population ${ }^{7}$. Based on familiarity and cost, metformin is widely used for this purpose in women with $\mathrm{PCOS}^{20}$. Other drugs 
used include orlistat, inositol and liraglutide ${ }^{21-23}$; however, their comparative efficacy in women with PCOS is unknown. We have conducted the first systematic review and meta-analysis to compare the effects of these drugs on weight loss in women with PCOS and overweight/obesity.

\section{Methods}

We followed the Preferred Reporting Items for Systematic Reviews and Meta-Analyses (PRISMA) statement to conduct our systematic review ${ }^{24}$.

\section{Study eligibility criteria}

We included studies that 1) used a randomised, parallel group trial design; 2) were conducted in the following settings: clinic, hospital, medical centre or community; 3) recruited adults/adolescents who have obesity/overweight with PCOS; and 4) included placebo or metformin as the control arm. Where studies reported similar or overlapping data, only the latest or those with a larger sample size were considered for this review. The outcomes of interest included BMI, weight, waist circumference and waist-to-hip ratio (WHR).

\section{Literature search methodology}

We searched the following databases for published articles and conference abstracts and proceedings in consultation with a search methodologist: MEDLINE (Ovid), 
EMBASE (Ovid), PubMed and the Cochrane Central Register of Controlled trials (CENTRAL). The search was performed in July 2017. No language or date restrictions were employed. Qualified librarians were consulted for the search strategy. Medical Subject Heading (MeSH) terms included key words defining PCOS ('polycystic ovary syndrome', 'Stein-Leventhal syndrome') and the relevant treatments of interest ('metformin', 'liraglutide', 'orlistat', 'inositol'). The full review protocol was registered with PROSPERO under registration number CRD 42017076625.

\section{Study selection}

After a primary screening of titles and abstracts (by F.W., Y.W., Y.Z and F.Q.), the full texts of all potentially eligible studies were retrieved. Three review authors (F.W., Y.W., and Y.Z.) independently examined and selected the eligible articles in compliance with the inclusion criteria for the current review. Study investigators were contacted if clarification regarding details of the study was needed. Disagreement with respect to study eligibility was resolved via discussion with the other reviewer (F.Q.). The selection process is presented in a PRISMA diagram.

\section{Data extraction}

Two review authors (F.W. and Y.W.) independently extracted the data from each eligible study. Any disagreements were resolved by discussion with a third review author (F.Q.). Data retrieved included characteristics of study, subjects and 
interventions, as well as outcome measures. Authors were contacted for additional or missing information as required.

\section{Risk of bias}

The risk of bias of the included studies was assessed independently by two authors (F.W. and Y.Z.) using the Cochrane Risk of Bias Tool 2.0 with respect to the following aspects: the randomisation process, deviations from intended interventions, missing outcome data, measurement of the outcome and selection of the reported results. Any discrepancies were resolved among the authors to reach a consensus.

\section{Statistical analysis}

We extracted point estimates of relevant means and standard deviations from individual studies. This includes mean difference and its associated standard deviation (SD) between the comparison groups after the experiment. If the SD associated with the mean difference was not reported, then a 95\% confidence interval $(\mathrm{CI})$ was used for the computation of SD. Moreover, if the mean difference and associated SD between the comparison groups was not reported, we extracted the arm-specific mean and SD both before and after the treatment to calculate these quantities. The formula for computing the mean change and its associated SD for a specific arm referred to Dias et al. ${ }^{25}$. The mean of the comparison groups was assumed to follow a normal distribution and, therefore, the calculation of mean difference (and associated SD) was 
based on the subtraction of two independent normal distributions ${ }^{26}$. If the equal variance assumption is valid (i.e., the variances of the pairwise groups were comparable), the pooled SD was calculated as $S_{\text {pooled }}=\sqrt{\frac{S_{1}^{2}\left(n_{1}-1\right)+S_{2}^{2}\left(n_{2}-1\right)}{n_{1}+n_{2}-2}}$, where $\mathrm{n}_{1}, \mathrm{n}_{2}$ are the sample size for the two arms and $\mathrm{S}_{1}, \mathrm{~S}_{2}$ are the arm-specific SDs; if the equal variance assumption does not hold, the following formula was applied to compute the pooled SD: $S_{\text {pooled }}=\sqrt{\frac{S_{1}^{2}}{n_{1}}+\frac{S_{2}^{2}}{n_{2}}}$.

A network meta-analysis ${ }^{25}$ was performed to simultaneously compare the efficacy of different treatment options for managing weight (i.e., BMI, absolute weight change) and waist circumference (i.e., absolute waist change). The purpose of a network metaanalysis is to combine results from direct and indirect comparisons of treatment effect in a single analysis. For example, an estimated difference of treatment effect between drug $\mathrm{A}$ and drug $\mathrm{C}$ can be obtained by comparing trials of drug $\mathrm{A}$ versus $\mathrm{B}$ with that of drug $B$ versus $C$. The network was graphed to illustrate the pairwise comparisons between different treatment options. We assessed both the heterogeneity within the trial and the inconsistency between trials using the $\mathrm{I}^{2}$ statistic and $\mathrm{p}$-value. A standard random-effects meta-analysis was conducted to compare the efficacy of metformin and placebo in terms of change in WHR as the number of eligible studies for this outcome of interest is not enough to construct a network.

We used standard meta-analyses with a random-effects model to examine any heterogeneity that may exist in each of the pairwise treatment comparisons (e.g. metformin versus placebo, orlistat versus metformin). The statistical heterogeneity was 
assessed with the $I^{2}$ statistic and $p$-value. In terms of inconsistency, heat plots were provided to visualise whether there was disagreement between direct and indirect comparisons $^{27,28}$.

Subgroup analysis was performed for studies that reported an outcome of interest (i.e., BMI, weight and waist circumference) with the same treatment duration (12 weeks or 24 weeks). A network meta-analysis was used for outcomes reported at 12 weeks (since more than two treatments were compared) whereas a standard meta-analysis was applied for outcomes reported at 24 weeks (only metformin and placebo were compared).

A funnel plot and a regression test that detected any asymmetry of the funnel plot were used to examine publication bias for standard meta-analysis. The $p$-value associated with the Egger's test statistic was also reported.

Sensitivity analysis was conducted to explore the effect of the summary estimate by excluding studies that were considered to result in high heterogeneity (i.e., $I^{2}>50 \%$ ) in the pairwise standard meta-analysis.

All the analyses were performed in R using the packages 'netmeta' (used for network meta-analysis) ${ }^{29}$ and 'metafor' (used for standard meta-analysis) ${ }^{30}$.

\section{Results}

\section{Search results}


The search strategy yielded 4307 studies; however, 3848 studies were excluded because it was clear from the title or abstract that they did not fulfil the selection criteria. After full-text review of 459 potential studies, we further excluded 15 duplicates, 318 studies not meeting the selection criteria and 100 studies due to lack of essential data (e.g., mean and SD of the change of BMI, body weight, waist circumference or WHR between before and after interventions cannot be retrieved or computed). A total number of 26 studies were included for the systemic review, among which three studies were excluded because different preparations of inositol were examined. This resulted in 23 eligible studies for the meta-analysis. Details of the literature search and selection process are shown in Fig. 1.

\section{Characteristics of included studies}

Table 1 presents characteristics of included studies. Trials were performed in European ${ }^{31-46}$, American $^{47-52}$ and $A \operatorname{sian}^{53-56}$ countries. Participants in these trials were recruited from outpatient clinics, hospitals or medical centres and were diagnosed with PCOS according to either the National Institutes of Health $(\mathrm{NIH})$ or Rotterdam criteria. Participants aged 18 years to menopause were included in 24 studies, and two studies examined the effect of metformin on weight reduction in adolescent women ${ }^{49,50}$. Apart from two studies where liraglutide $1.8 \mathrm{mg} /$ day for 26 weeks and $3.0 \mathrm{mg} /$ day for 12 weeks were applied ${ }^{40,41}, 1.2 \mathrm{mg} /$ day was used for 12 weeks in the remaining five studies examining the efficacy of liraglutide. The use of liraglutide1.2 mg/day in 
combination with metformin $2.0 \mathrm{~g} /$ day was identified in four trials ${ }^{36,39,40,42}$. Participants received a dosage of metformin ranging from 1.5 to $2.0 \mathrm{~g} /$ day for 12 or 24 weeks. The dose of orlistat was $360 \mathrm{mg} /$ day and the duration of treatment was 12 weeks in all relevant studies. In addition, over $50 \%$ of the trials examining metformin and orlistat recommended lifestyle modifications such as dieting and exercise, whereas the corresponding figure was only $28.6 \%$ for trials examining liraglutide. For studies included in the meta-analysis, the dropout rate was $9.2 \%(12 / 130)$ for liraglutide alone, $6.0 \%(3 / 50)$ for liraglutide + metformin, $12.8 \%$ (47/366) for metformin alone, $5.4 \%$ (7/130) for orlistat and $9.5 \%$ (30/315) for placebo. Two studies did not report the dropout rate in each arm. Three studies compared the effects of inositol; however, Nestler et al. ${ }^{52}$ studied D-chiro-inositol, Gerli et al. ${ }^{45}$ studied myo-inositol, and Nordio et al. ${ }^{46}$ studied myo-inositol alone or in combination with D-chiro-inositol. The results of these studies could therefore not be combined for meta-analysis. None of the trials assessing inositol included lifestyle modification.

\section{Results from risk of bias assessment}

Using the RoB 2.0 tool $^{57}$, of 26 randomised controlled trials (RCTs), there were 16 (61.5\%) RCTs with low risk of bias arising from the randomisation process, 23 (88.5\%) RCTs with low risk of bias due to deviations from intended interventions and $19(73.1 \%)$ RCTs with low risk of bias due to missing outcome data; all assessed RCTs had low risk of bias in measurement of the outcome, and 24 (92.3\%) RCTs had low risk of bias in selection of the reported results. RoB could not be assessed for Jensterle et al. 
$(2017 b)^{42}$ because of insufficient information. Low risk, some concerns and high risk

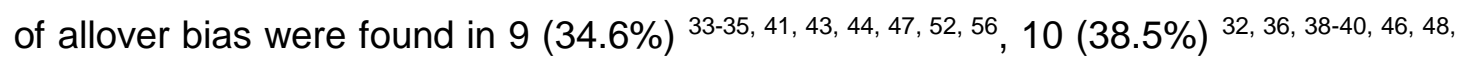
49, 53, 55 and $6(23.1 \%){ }^{31,37,45,50,51,54} \mathrm{RCTs}$. The high risk of allover bias originated from missing outcome data $31,37,45,50,51$ and selection of the reported results ${ }^{54}$. Table 2 shows results from the risk of bias assessment.

\section{Efficacy outcomes}

The network of eligible comparison for efficacy is presented in Fig. 2. Whilst metformin was directly compared with all the other treatments, the comparisons between the remaining four treatments (i.e., liraglutide alone, the combination of liraglutide and metformin, orlistat and placebo) were connected either directly or indirectly by at least one trial.

Our network meta-analysis included 23 RCTs with a total number of 941 participants. Apart from four trials where a combination of liraglutide and metformin (60 women) was evaluated, the remaining trials offered a single drug as an intervention, including liraglutide ( 6 trials, of which 5 used a dose below the 3 mg licensed dose for obesity treatment, 118 women), metformin ( 19 trials, 338 women), orlistat ( 5 trials, 123 women) and placebo (14 trials, 302 women).

Compared with the placebo, all the interventions (liraglutide, metformin, orlistat, and liraglutide in combination with metformin) resulted in a significant reduction in BMI in women with PCOS and overweight/obesity (Fig. 3A). The efficacy of all the treatments 
was compared pairwise (Table 3). Using subgroup analysis, only orlistat significantly reduced BMI after 12 weeks of treatment (Fig. 3B and Table 3). Similarly, there was no significant impact of metformin on BMI reduction after 24 weeks of treatment (Fig. 3C). The funnel plot showed minor asymmetry (Fig. S1A).

Compared with the placebo, all the interventions, liraglutide, metformin, orlistat and the combination of liraglutide and metformin, led to a significant decrease in weight (Fig. 4A). The magnitude of weight reduction differed significantly among drugs (in descending order): liraglutide alone, orlistat and metformin alone (Table 4). Liraglutide alone was more effective than liraglutide and metformin combined. This may reflect the higher liraglutide dose in studies of monotherapy than in those where it was used with metformin. At 12 weeks, only orlistat had a significant impact on BMI, whilst liraglutide alone and orlistat had a significant effect on weight (Fig. 4B and Table 4). The results from 24 weeks suggested that metformin was superior to the placebo (Fig. 4C). Funnel plot analysis indicated no publication bias (Fig. S1B).

The results for waist circumference (Fig. 5 and Table 5) were mainly consistent with those for BMI and weight: liraglutide alone, liraglutide in combination with metformin and metformin alone led to a significant decrease in the waist circumference of women with PCOS and overweight/obesity (Fig. 5A). Liraglutide alone was more effective than metformin (Table 5). The reduction in waist circumference was not significant compared with the placebo for all treatments at 12 weeks (Fig. 5B and Table 5). Waist 
circumference was significantly reduced after 24 weeks of metformin therapy (Fig. 5C). There was minor publication bias (Fig. S1C).

Data for WHR were only available for metformin; metformin was not found to be superior to the placebo, based on results of all included studies (Fig. 6A) or only 24week studies (Fig. 6B). Again, minor publication bias was present (Fig. S1D and E). Pairwise meta-analysis showed significant heterogeneity $\left(I^{2}=71 \%\right)$ for the direct comparison between metformin and the placebo (results for waist circumference based on all the included studies), but there was no heterogeneity $\left(I^{2}=0\right)$ or moderate heterogeneity $\left(I^{2}<25 \%\right)$ for all the remaining comparisons. No significant inconsistency was identified from our assessment based on the heat plots (Fig. S2).

\section{Sensitivity analysis}

One study ${ }^{49}$ was excluded from the sensitivity analysis for waist circumference because it was the leading contributor to heterogeneity in the direct comparison between metformin and placebo. The results of the network meta-analysis after removing this study (Fig. S3 and Table S1) were consistent with the previous results for waist circumference (Fig. 5A).

Four trials ${ }^{31,48-50}$ were excluded in the sensitivity analysis for BMI, weight and waist circumference because the sample size was less than 10 per arm. The results of the network meta-analysis after removing these trials (Fig. S4A-C and Table S2A-C) were 
consistent with the previous results for BMI, weight and waist circumference (Fig. 3A, $4 \mathrm{~A}$ and $5 \mathrm{~A})$.

Two studies ${ }^{49,50}$ were excluded from the sensitivity analysis for BMI and waist circumference because they included adolescents. However, none of these studies assessed weight loss. The results of the network meta-analysis after removing these studies (Fig. S5A and B; Table S3A and B) were consistent with the previous results for $\mathrm{BMI}$ and waist circumference (Fig. 3A and $5 \mathrm{~A}$ ).

\section{Discussion}

As far as we are aware, this is the first meta-analysis to compare the effects of the treatments currently used to induce weight loss in women with PCOS and overweight/obesity. The findings have important implications for clinical practice. We used network meta-analysis to simultaneously compare the efficacy of a range of interventions because a network allows for indirect comparisons of treatments which are not studied in a head-to-head fashion. The findings for the different treatments were consistent for all anthropometric measures: BMI, weight, waist circumference and WHR.

Although this analysis was directed at pharmacological treatment, a hypocaloric diet and physical activity remain the first line treatments for weight loss in women with $\mathrm{PCOS}^{58}$. Aerobic physical activity of moderate intensity for 90 minutes per week is recommended for patients with $\mathrm{PCOS}^{59}$, and 5 to $15 \%$ weight loss or more is 
considered to improve clinical symptoms ${ }^{60}$. However, lifestyle interventions seem not to be effective in providing lasting weight loss success ${ }^{61}$. In addition, there is heterogeneity in the responsiveness to long-term lifestyle intervention in women with overweight/ obesity and PCOS ${ }^{62}$. Thus, more aggressive treatments, such as weightloss medication, should be added to patients who fail to respond to lifestyle intervention ${ }^{63}$.

For change in BMI and weight, all the drugs that qualified be evaluated in metaanalysis (liraglutide, metformin (alone and in combination) and orlistat) were superior to the placebo. There were, however, significant differences in the efficacy of these drugs; for weight loss, treatment with liraglutide was superior to all other treatments and orlistat was superior to metformin based on the data of all the included studies. It was not possible to calculate the mean percentage weight loss for the three drugs, but the results from the included studies show a relatively modest effect: after 12 weeks of therapy, orlistat $(360 \mathrm{mg})-1.02 \pm 0.9 \%^{43}$ to $-6.37 \%{ }^{56}$ and metformin $(1.5 \mathrm{~g})-4.69 \pm$ $1.3 \%{ }^{43}$ to $-5.04 \pm 0.67 \%{ }^{55}$. With liraglutide monotherapy, 16,42 and $57 \%$ of patients achieved weight loss of at least $5 \%$ in doses of $1.2,1.2$ and 3 mg daily, respectively ${ }^{36}$, $39,40$.

With the exception of a few outcomes confined to orlistat and liraglutide, 12 weeks of treatment is ineffective, so treatment should be continued for at least 24 weeks and probably much longer as the available evidence suggests that lost weight is regained when treatment is stopped. However, orlistat is only licensed for three years use. 
Liraglutide, a glucagon-like peptide-1 receptor agonist, was developed to treat type II diabetes ${ }^{15}$ but weight loss has been well described as an additional benefit, which prompted the manufacturer to develop a higher dose formulation specifically for this indication ${ }^{15}$. Liraglutide is also used by some doctors to treat women who have obesity/overweight with PCOS. Orlistat, which has an established safety profile ${ }^{64}$, is a reversible inhibitor of gastric and pancreatic lipases. As it is generally well tolerated, orlistat is an option for treatment of patients affected by obesity with or without type II diabetes and also has a role in the management of patients with metabolic syndrome and obesity, associated comorbidities or concomitant disorders ${ }^{65}$. Myo-inositol and Dchiro-inositol are increasingly used by doctors and by self-medication to treat the symptoms of PCOS. These compounds improve insulin resistance, reduce serum androgen levels and ameliorate many features of metabolic syndrome ${ }^{66}$. Unfortunately, we found only single studies of each isomer so these drugs could not be evaluated in this meta-analysis.

Some other drugs, such as bupropion/naltrexone, lorcaserin, and topiramate/ phentermine, are also widely used in the treatment of obesity. They aid in weight loss mainly by reducing food intake through different mechanisms ${ }^{60}$. Topiramate/phentermine seems to have a higher percentage of weight loss when compared with the other two, and there is no difference between lorcaserin and bupropion/naltrexone ${ }^{63}$. However, tpiramate/phentermine have shown risks of teratogenicity and psychiatric disturbances ${ }^{67}$, while the other two have more 
acceptable safety profiles. Unfortunately, these anti-obesity drugs have not been reported in women with PCOS.

Although BMl is the most widely used measure of obesity, there is increasing evidence that waist circumference is more closely associated with insulin resistance $(\mathrm{IR})^{2,68}$. Since waist circumference is positively correlated with the visceral adiposity index ${ }^{69}$ and $98 \%$ of women with PCOS and metabolic syndrome have an abnormal waist circumference ${ }^{70}$, this measurement is particularly relevant to the present study. In support of this, waist circumference was found to be the simplest method of clinical screening for IR in women with hirsutism ${ }^{68}$. None of the drugs evaluated had a significant effect on waist circumference after 12 weeks. However, based on data of all the included studies, liraglutide and metformin (alone or in combination) were superior to the placebo. Data was not available for 24 weeks of therapy with the other treatments, but network analysis showed that liraglutide was superior to metformin after combining all the included studies.

The results of this analysis provide the first comparison of the drugs currently used to induce weight loss in women with PCOS and overweight/obesity. Effective therapy is urgently needed in view of the high prevalence of obesity in women with this syndrome, and its adverse effects on symptoms (including infertility) and increased risk of long term morbidity(e.g. diabetes and coronary heart disease) $)^{2,12}$. Although all the drugs were superior to the placebo to induce weight loss, liraglutide was the most effective. Liraglutide and metformin (alone or in combination) reduced waist circumference but, 
again, liraglutide was superior. Orlistat was superior to metformin in terms of weight loss but may be seen as inferior as it was not shown to reduce waist circumference. Our results also suggest that 12 weeks of treatment was not effective. Finally, the relative efficacy of the three drugs must be judged against their cost, with liraglutide being 100-fold and orlistat 10-fold more expensive than metformin. As metformin, liraglutide and orlistat were being assessed for their effects on obesity, rather than overweight or symptoms of PCOS, lifestyle modification is an essential adjunct to pharmacologic therapy in this situation.

The present study has some limitations. Firstly, publication bias may exist given that studies with significant results are more likely to be published, although we have attempted to address this issue by retrieving all the available studies and a funnel plot was used to assess any bias that may have arisen from this source. Secondly, we could not perform an analysis for the different inositol isomers. Data for the effects of 24 weeks of treatment were only available for metformin. Two studies ${ }^{49,}{ }^{50}$ were confined to treating adolescent patients with PCOS. Finally, there was high heterogeneity for the comparison between metformin and placebo with respect to waist circumference, which was considered to be caused by one study ${ }^{49}$ where an increase in waist circumference was observed after 24 weeks. This may reflect the sample size as only six participants were included in the intervention arm. Sample size may also explain the wide Cls for all the anthropometric measures for the liraglutide/metformin combination. As our initiative is to compare more than 2 drugs simultaneously (can 
only be achieved through constructing a network meta-analysis) to give clinicians a clue of medication options to induce weight loss in women with PCOS and overweight/obesity, in our meta-analysis, we included all the studies that met the inclusion criteria. Admittedly, small studies are likely to contribute to significant amount of bias as there is more uncertainty in estimates and the results are more likely to be spontaneous. Moreover, adolescents may have different hormonal and metabolic profile as well as behavior compared with adults. To deal with this issue, we have conducted sensitivity analysis by removing the studies with small sample size (less than 10 in each arm) and the studies with adolescents. We did not find any significant changes on the conclusions of our findings (shown as Supplementary materials).

This study focused on the effects of the currently available drugs that are most commonly used to treat women with PCOS and obesity. However, new and possibly more effective anti-obesity drugs such as semaglutide (a once weekly GLP-1 agonist which induces weight loss of around $13.8 \%)^{71}$, bupropion/naltrexone and lorcaserin are now available, and these may have an important role in treating women with PCOS.

\section{Conclusion}

The results of this study provide evidence that the three treatments with data suitable for analysis (liraglutide, metformin and orlistat) reduce BMI and weight in women with PCOS and overweight/obesity. Although effective, they are adjunct to, not a replacement for, a low energy diet and increased physical activity. Using network 
analysis, we found that liraglutide was the most effective and metformin the least effective intervention to lower BMI and weight. In view of the fact that the obesity that affects women with PCOS is predominantly central and this visceral fat is associated with anovulation, diabetes and cardiovascular morbidity, we also assessed the effects of these drugs on waist circumference. For this endpoint, liraglutide was again more effective than metformin, but we did not find a statistically significant effect for orlistat. In clinical practice, effectiveness has to be weighed against cost. We have not performed an economic evaluation, but on the basis that the cost of liraglutide is approximately 100 -fold more expensive than metformin and 10 -fold more expensive than orlistat, it seems unrealistic to suggest widespread use of this drug for this indication. The relative cost effectiveness of orlistat and metformin is less obvious, with orlistat having the benefit of greater weight loss but the possible disadvantage of a lesser effect on visceral fat. In the absence of a healthy economic evaluation, the results of our analysis support the use of either metformin or orlistat to induce weight loss in women with PCOS and overweight/obesity. However, the conclusion may seem limited due to the insufficient evidences to date to guide policy recommendations.

\section{Conflict of interest}

None declared. 


\section{Acknowledgement}

Authors would like to acknowledge Jennifer Ford, evidence services librarian at the

Royal Free Hospital Medical Library of University College London, for her assistance

with literature search.

\section{Funding}

This study was supported by the National Natural Science Foundation of China (81401167 to F.W.), the Zhejiang Province Science Foundation for Distinguished Young Scholars (LR16H040001 to F.Q.), the Medical and Health General Project of Zhejiang Province, China (2015KYA122 to F.W.), the State Scholarship Fund of China Scholarship Council (201606325016 to F.W.) and the National Institute for Health Research (RP-2015-06-005 to R.L.B.).

\section{References}

1. World Health Organization (WHO). Obesity, retrieved on 10 September 2017. http://www.euro.who.int/en/health-topics/noncommunicable-diseases/obesity. 2. Lim SS, Davies MJ, Norman RJ, Moran LJ. Overweight, obesity and central obesity in women with polycystic ovary syndrome: a systematic review and meta-analysis. Hum Reprod Update 2012; 18: 618-637.

3. Dumesic DA, Oberfield SE, Stener-Victorin E, Marshall JC, Laven JS, Legro RS. Scientific Statement on the Diagnostic Criteria, Epidemiology, Pathophysiology, and Molecular Genetics of Polycystic Ovary Syndrome. Endocr Rev 2015; 36: 487525.

4. Stein IF, Leventhal ML. Amenorrhea associated with bilateral polycystic ovaries. Am J Obstet Gynecol 1935; 29: 181-191.

5. Legro RS. Obesity and PCOS: implications for diagnosis and treatment. Semin 
Reprod Med 2012; 30: 496-506.

6. Nestler JE, Jakubowicz DJ, de Vargas AF, Brik C, Quintero N, Medina F. Insulin stimulates testosterone biosynthesis by human thecal cells from women with polycystic ovary syndrome by activating its own receptor and using inositolglycan mediators as the signal transduction system. J Clin Endocrinol Metab 1998; 83: 2001-2005.

7. McCartney CR, Marshall JC. CLINICAL PRACTICE. Polycystic Ovary Syndrome. $N$ Engl J Med 2016; 375: 54-64.

8. Targher G. Polycystic Ovary Syndrome. N Engl J Med 2016; 375: 1397-1398.

9. Barry JA, Azizia MM, Hardiman PJ. Risk of endometrial, ovarian and breast cancer in women with polycystic ovary syndrome: a systematic review and metaanalysis. Hum Reprod Update 2014; 20: 748-758.

10. Al-Azemi M, Omu FE, Omu AE. The effect of obesity on the outcome of infertility management in women with polycystic ovary syndrome. Arch Gynecol Obstet 2004; 270: 205-210.

11. Bagir GS, Bakiner OS, Bozkirli E, Cavlak G, Serinsoz H, Ertorer ME. Body Mass Index below Obesity Threshold Implies Similar Cardiovascular Risk among Various Polycystic Ovary Syndrome Phenotypes. Med Princ Pract 2016; 25: 61-66. 12. Lim SS, Norman RJ, Davies MJ, Moran LJ. The effect of obesity on polycystic ovary syndrome: a systematic review and meta-analysis. Obes Rev 2013; 14: 95109.

13. Pandey S, Pandey S, Maheshwari A, Bhattacharya S. The impact of female obesity on the outcome of fertility treatment. J Hum Reprod Sci 2010; 3: 62-67.

14. Teede HJ, Misso ML, Deeks AA, et al. Assessment and management of polycystic ovary syndrome: summary of an evidence-based guideline. Med J Aust 2011; 195: S65-112.

15. Moran LJ, Hutchison SK, Norman RJ, Teede HJ. Lifestyle changes in women with polycystic ovary syndrome. Cochrane Database Syst Rev 2011: CD007506.

16. Naderpoor N, Shorakae S, de Courten B, Misso ML, Moran LJ, Teede HJ. Metformin and lifestyle modification in polycystic ovary syndrome: systematic review and meta-analysis. Hum Reprod Update 2015; 21: 560-574.

17. Skubleny D, Switzer NJ, Gill RS, et al. The Impact of Bariatric Surgery on Polycystic Ovary Syndrome: a Systematic Review and Meta-analysis. Obes Surg 2016; 26: 169-176.

18. Jensterle M, Kocjan T, Kravos NA, Pfeifer M, Janez A. Short-term intervention with liraglutide improved eating behavior in obese women with polycystic ovary syndrome. Endocr Res 2015; 40: 133-138.

19. Vosnakis C, Georgopoulos NA, Rousso D, et al. Diet, physical exercise and Orlistat administration increase serum anti-Mullerian hormone (AMH) levels in women with polycystic ovary syndrome (PCOS). Gynecol Endocrinol 2013; 29: 242-245.

20. Harborne L, Fleming R, Lyall H, Norman J, Sattar N. Descriptive review of the 
evidence for the use of metformin in polycystic ovary syndrome. Lancet 2003; 361: 1894-1901.

21. Norman RJ, Dewailly D, Legro RS, Hickey TE. Polycystic ovary syndrome. Lancet 2007; 370: 685-697.

22. Pundir J, Psaroudakis D, Savnur P, et al. Inositol treatment of anovulation in women with polycystic ovary syndrome: a meta-analysis of randomised trials. BJOG 2017; 125: 299-308.

23. Panidis D, Farmakiotis D, Rousso D, Kourtis A, Katsikis I, Krassas G. Obesity, weight loss, and the polycystic ovary syndrome: effect of treatment with diet and orlistat for 24 weeks on insulin resistance and androgen levels. Fertil Steril 2008; 89: 899-906.

24. Liberati A, Altman DG, Tetzlaff J, et al. The PRISMA Statement for Reporting Systematic Reviews and Meta-Analyses of Studies That Evaluate Health Care Interventions: Explanation and Elaboration. Plos Med 2009; 6: e1000100.

25. Dias S, Welton NJ, Sutton AJ, Ades AE. A Generalised Linear Modelling

Framework for Pairwise and Network Meta-Analysis of Randomised Controlled

Trials. National Institute for Health and Care Excellence (NICE): London 2014.

26. Rosenblad A. Introduction to Meta-Analysis by Michael Borenstein, Larry V. Hedges, Julian P.T. Higgins, Hannah R. Rothstein. Int Stat Rev 2009; 77: 478-479.

27. Krahn U, Binder $\mathrm{H}$, Konig J. A graphical tool for locating inconsistency in network meta-analyses. BMC Med Res Methodol 2013; 13: 35.

28. Krahn U, Binder H, Konig J. Visualizing inconsistency in network meta-analysis by independent path decomposition. BMC Med Res Methodol 2014; 14: 131.

29. Rücker G, Schwarzer G, Krahn U, König J. netmeta: Network Meta-Analysis using Frequentist Methods. R package version 08-0 2015.

30. Viechtbauer W. Conducting Meta-Analyses in R with the metafor Package.J Stat Softw 2010; 36: 1-48.

31. Pasquali R, Gambineri A, Biscotti D, et al. Effect of long-term treatment with metformin added to hypocaloric diet on body composition, fat distribution, and androgen and insulin levels in abdominally obese women with and without the polycystic ovary syndrome. J Clin Endocrinol Metab 2000; 85: 2767-2774.

32. Gambineri A, Pelusi C, Genghini S, et al. Effect of flutamide and metformin administered alone or in combination in dieting obese women with polycystic ovary syndrome. Clin Endocrinol (Oxf) 2004; 60: 241-249.

33. Gambineri A, Patton L, Vaccina A, et al. Treatment with flutamide, metformin, and their combination added to a hypocaloric diet in overweight-obese women with polycystic ovary syndrome: a randomized, 12-month, placebo-controlled study. J Clin Endocrinol Metab 2006; 91: 3970-3980.

34. Tang T, Glanville J, Hayden CJ, White D, Barth JH, Balen AH. Combined lifestyle modification and metformin in obese patients with polycystic ovary syndrome. A randomized, placebo-controlled, double-blind multicentre study. Hum Reprod 2006; 21: 80-89. 
35. Sova H, Puistola U, Morin-Papunen L, Karihtala P. Metformin decreases serum 8-hydroxy-2'-deoxyguanosine levels in polycystic ovary syndrome. Fertil Steril 2013; 99: 593-598.

36. Jensterle Sever M, Kocjan T, Pfeifer M, Kravos NA, Janez A. Short-term combined treatment with liraglutide and metformin leads to significant weight loss in obese women with polycystic ovary syndrome and previous poor response to metformin. Eur J Endocrinol 2014; 170: 451-459.

37. Jensterle M, Kravos NA, Pfeifer M, Kocjan T, Janez A. A 12-week treatment with the long-acting glucagon-like peptide 1 receptor agonist liraglutide leads to significant weight loss in a subset of obese women with newly diagnosed polycystic ovary syndrome. Hormones (Athens) 2015; 14: 81-90.

38. Jensterle M, Salamun V, Kocjan T, Vrtacnik Bokal E, Janez A. Short term monotherapy with GLP-1 receptor agonist liraglutide or PDE 4 inhibitor roflumilast is superior to metformin in weight loss in obese PCOS women: a pilot randomized study. J Ovarian Res 2015; 8: 32.

39. Jensterle M, Goricar K, Janez A. Metformin as an initial adjunct to low-dose liraglutide enhances the weight-decreasing potential of liraglutide in obese polycystic ovary syndrome: Randomized control study. Exp Ther Med 2016; 11: 1194-1200.

40. Jensterle M, Kravos NA, Goricar K, Janez A. Short-term effectiveness of low dose liraglutide in combination with metformin versus high dose liraglutide alone in treatment of obese PCOS: randomized trial. BMC Endocr Disord 2017; 17: 5.

41. Nylander M, Frossing S, Kistorp C, Faber J, Skouby SO. Liraglutide in polycystic ovary syndrome: a randomized trial, investigating effects on thrombogenic potential. Endocr Connect 2017; 6: 89-99.

42. Jensterle M, Salamun V, Bokal EV, Janez A. Short-term intervention with liraglutide and metformin increased fertility potential in a subset of obese PCOS proceeding in vitro sterilisation. Diabetes 2017; 66: A561.

43. Jayagopal V, Kilpatrick ES, Holding S, E. JP, Atkin SL. Orlistat is as beneficial as metformin in the treatment of polycystic ovarian syndrome. J Clin Endocrinol Metab 2005; 90: 729-733.

44. Cho LW, Kilpatrick ES, Keevil BG, Coady AM, Atkin SL. Effect of metformin, orlistat and pioglitazone treatment on mean insulin resistance and its biological variability in polycystic ovary syndrome. Clin Endocrinol (Oxf) 2009; 70: 233-237. 45. Gerli S, Mignosa M, Di Renzo GC. Effects of inositol on ovarian function and metabolic factors in women with PCOS: a randomized double blind placebocontrolled trial. Eur Rev Med Pharmacol Sci 2003; 7: 151-159.

46. Nordio M, Proietti E. The combined therapy with myo-inositol and D-chiroinositol reduces the risk of metabolic disease in PCOS overweight patients compared to myo-inositol supplementation alone. Eur Rev Med Pharmacol Sci 2012; 16: 575-581.

47. Chou KH, von Eye Corleta H, Capp E, Spritzer PM. Clinical, metabolic and 
endocrine parameters in response to metformin in obese women with polycystic ovary syndrome: a randomized, double-blind and placebo-controlled trial. Horm Metab Res 2003; 35: 86-91.

48. Maciel GA, Soares Junior JM, Alves da Motta EL, Abi Haidar M, de Lima GR, Baracat EC. Nonobese women with polycystic ovary syndrome respond better than obese women to treatment with metformin. Fertil Steril 2004; 81: 355-360.

49. Hoeger K, Davidson K, Kochman L, Cherry T, Kopin L, Guzick DS. The impact of metformin, oral contraceptives, and lifestyle modification on polycystic ovary syndrome in obese adolescent women in two randomized, placebo-controlled clinical trials. J Clin Endocrinol Metab 2008; 93: 4299-4306.

50. Ladson G, Dodson WC, Sweet SD, et al. Effects of metformin in adolescents with polycystic ovary syndrome undertaking lifestyle therapy: a pilot randomized double-blind study. Fertil Steril 2011; 95: 2595-2598.

51. Metwally M, Amer S, Li TC, Ledger WL. An RCT of metformin versus orlistat for the management of obese anovulatory women. Hum Reprod 2009; 24: 966-975.

52. Nestler JE, Jakubowicz DJ, Reamer P, Gunn RD, Allan G. Ovulatory and metabolic effects of D-chiro-inositol in the polycystic ovary syndrome. N Engl J Med 1999; 340: 1314-1320.

53. Onalan G, Goktolga U, Ceyhan T, Bagis T, Onalan R, Pabuccu R. Predictive value of glucose-insulin ratio in PCOS and profile of women who will benefit from metformin therapy: obese, lean, hyper or normoinsulinemic? Eur J Obstet Gynecol Reprod Biol 2005; 123: 204-211.

54. Amiri M, Golsorkhtabaramiri M, Esmaeilzadeh S, et al. Effect of Metformin and Flutamide on Anthropometric Indices and Laboratory Tests in Obese/Overweight PCOS Women under Hypocaloric Diet. J Reprod Infertil 2014; 15: 205-213.

55. Ghandi S, Aflatoonian A, Tabibnejad N, Moghaddam MH. The effects of metformin or orlistat on obese women with polycystic ovary syndrome: a prospective randomized open-label study. J Assist Reprod Genet 2011; 28: 591-596. 56. Moini A, Kanani M, Kashani L, Hosseini R, Hosseini L. Effect of orlistat on weight loss, hormonal and metabolic profiles in women with polycystic ovarian syndrome: a randomized double-blind placebo-controlled trial. Endocrine 2015; 49: 286-289. 57. Higgins JPT, J.A.C. S, J. S, et al. A revised tool for assessing risk of bias in randomized trials In: Chandler J, McKenzie J, Boutron I, Welch V (eds). Cochrane Methods. Cochrane Database of Systematic Reviews 2016: Suppl 1.

58. van Baal PH, van den Berg M, Hoogenveen RT, Vijgen SM, Engelfriet PM. Costeffectiveness of a low-calorie diet and orlistat for obese persons: modeling longterm health gains through prevention of obesity-related chronic diseases. Value Health 2008; 11: 1033-1040.

59. Harrison CL, Lombard CB, Moran LJ, Teede HJ. Exercise therapy in polycystic ovary syndrome: a systematic review. Hum Reprod Update 2010; 17: 171-183.

60. Garvey WT, Mechanick JI, Brett EM, et al. American Association of Clinical Endocrinologists and American College of Endocrinology comprehensive clinical 
practice guidelines for medical care of patients with obesity. Endocr Pract 2016; 22: 1-203.

61. Lowe MR. Self - regulation of energy intake in the prevention and treatment of obesity: Is it feasible? Obes Res 2003; 11: S44-59.

62. Pasquali R, Gambineri A, Cavazza C, et al. Heterogeneity in the responsiveness to long-term lifestyle intervention and predictability in obese women with polycystic ovary syndrome. Eur J Endocrinol 2011; 164: 53-60.

63. Kumar RB, Aronne LJ. Efficacy comparison of medications approved for chronic weight management. Obesity 2015; 23: S4-7.

64. Halpern B, Halpern A. Safety assessment of FDA-approved (orlistat and lorcaserin) anti-obesity medications. Expert Opin Drug Saf 2015; 14: 305-315.

65. Henness S, Perry CM. Orlistat: a review of its use in the management of obesity. Drugs 2006; 66: 1625-1656.

66. Dinicola S, Chiu TT, Unfer V, Carlomagno G, Bizzarri M. The rationale of the myoinositol and D-chiro-inositol combined treatment for polycystic ovary syndrome. J Clin Pharmacol 2014; 54: 1079-1092.

67. George M, Rajaram M, Shanmugam E. New and emerging drug molecules against obesity. J Cardiovasc Pharmacol Ther 2014; 19: 65-76.

68. Toscani M, Migliavacca R, Sisson de Castro JA, Spritzer PM. Estimation of truncal adiposity using waist circumference or the sum of trunk skinfolds: a pilot study for insulin resistance screening in hirsute patients with or without polycystic ovary syndrome. Metabolism 2007; 56: 992-997.

69. Durmus U, Duran C, Ecirli S. Visceral adiposity index levels in overweight and/or obese, and non-obese patients with polycystic ovary syndrome and its relationship with metabolic and inflammatory parameters. J Endocrinol Invest 2017; 40: 487-497.

70. Glueck CJ, Papanna R, Wang P, Goldenberg N, Sieve-Smith L. Incidence and treatment of metabolic syndrome in newly referred women with confirmed polycystic ovarian syndrome. Metabolism 2003; 52: 908-915.

71. Bagsværd, Denmark. Novo Nordisk reports up to $13.8 \%$ weight loss in people with obesity receiving semaglutide in phase 2 trial, retrieved on 10 September 2017. http://www.novonordisk.com. 


\section{Table and Figure Legends}

Table 1: Characteristics of the studies included in the systematic review.

Table 2: Risk of bias assessment using RoB 2.0 tool.

Table 3: Network meta-analysis of efficacy of intervention options on body mass index (BMI) change. The efficacy estimate is located at the intersection of the columndefining treatment and the row-defining treatment. A mean difference below zero favours the column-defining treatment. Bold and underlined results are statistically significant.

Table 4: Network meta-analysis of efficacy of intervention options on weight change.The efficacy estimate is located at the intersection of the column-defining treatment and the row-defining treatment. A mean difference below zero favours the column-defining treatment. Bold and underlined results are statistically significant.

Table 5: Network meta-analysis of efficacy of intervention options on waist circumference change.The efficacy estimate is located at the intersection of the column-defining treatment and the row-defining treatment. A mean difference below zero favours the column-defining treatment. Bold and underlined results are statistically significant.

Figure 1: Preferred Reporting Items for Systematic Reviews and Meta-Analyses (PRISMA) flow diagram of study selection. Full-text articles included abstract only publications. 
Figure 2: Network of eligible comparisons for efficacy. The size of the circles is proportional to sample size, and the width of lines is proportional to the number of trials. LIRA: liraglutide, MET: metformin, ORL: orlistat, PBO: placebo.

Figure 3: Meta-analysis of efficacy of treatment options on body mass index (BMI) change compared with placebo. (A) Network meta-analysis of drug efficacy on the change in BMI (results based on combining all the included studies, i.e., 12 weeks and 24/26 weeks); (B) Network meta-analysis of drug efficacy on change in BMI (results for 12 weeks); (C) Standard meta-analysis comparing the efficacy of metformin with placebo on the change in $\mathrm{BMI}$ at 24 weeks. $\mathrm{PBO}$ : placebo, MD: mean difference, $\mathrm{Cl}$ : confidence interval, LIRA: liraglutide, MET: metformin, ORL: orlistat, N: sample size, RE: random effects.

Figure 4: Network meta-analysis of efficacy of treatment options on weight loss compared with the placebo. (A) Network meta-analysis of drug efficacy on weight loss (results based on combining all the included studies, i.e., 12/14 weeks and 24/26 weeks); (B) Network meta-analysis of drug efficacy on weight loss (results for 12 weeks); (C) Standard meta-analysis comparing the efficacy of metformin with placebo on weight loss at 24 weeks. PBO: placebo, MD: mean difference, $\mathrm{Cl}$ : confidence interval, LIRA: liraglutide, MET: metformin, ORL: orlistat, $N$ : sample size, RE: random effects.

Figure 5: Network meta-analysis of efficacy of treatment options on waist circumference change compared with placebo. (A) Network meta-analysis of drug 
efficacy on the change in waist circumference (results based on combining all the included studies, i.e., 12 weeks and 24/26 weeks); (B) Network meta-analysis of drug efficacy on change in waist circumference (results for 12 weeks); (C) Standard metaanalysis comparing the efficacy of metformin with placebo on the change in waist circumference at 24 weeks. PBO: placebo, MD: mean difference, $\mathrm{Cl}$ : confidence interval, LIRA: liraglutide, MET: metformin, ORL: orlistat, N: sample size, RE: random effects.

Figure 6: Role of metformin in waist-to-hip ratio (WHR) change. (A) Standard meta-analysis of efficacy of metformin on WHR change compared with placebo (results based on combining all the included studies, i.e., 12 weeks and 24 weeks). (B) Standard meta-analysis of metformin efficacy on change in WHR (results for 24 weeks). MD: mean difference, $\mathrm{Cl}$ : confidence interval, RE: random effects, MET: metformin, PBO: placebo, N: sample size. 